\title{
EDITORIAL
}

\section{CESÁREAS: POCA PERO SUFICIENTE INFORMACIÓN PARA PONER COTO A LA INADECUACIÓN}

\section{Carlos Campillo Artero}

Servicio de Salud de las Islas Baleares. Palma de Mallorca.

Hay estipulaciones cuya interpretación y puesta en práctica algo caprichosas confunden el intelecto colectivo a largo plazo. Dos ejemplos: la fijación del umbral de significación estadística, introducido por Pearson y difundido ampliamente por Fisher, en 0,05 y de la tasa máxima aceptable de cesáreas en $15 \%{ }^{1,2}$.

Aunque el ascenso paulatino durante décadas de las tasas brutas de cesáreas y sus comparaciones entre hospitales sugieren sobreindicación de esta intervención (inadecuación), conocemos los peligros que encierran tanto las conclusiones que se extraigan como, sobre todo, las decisiones que se tomen y las acciones que se emprendan a partir de ellas. Dado el virtual estancamiento de los sistemas de información poblacionales en las tasas brutas de cesáreas, debe insistirse en que, sin comprobar si son distintas de las debidamente ajustadas por riesgo (entre otras variables, por edad de la madre, edad gestacional, paridad, cesárea previa, comorbilidad) puede ser temerario y, en el mejor de los casos, estéril impulsar intervenciones carentes de especificidad de objetivo (tanto como lo ha sido vincular la productividad variable de los contratos de gestión con el mantenimiento por debajo de 15 del porcentaje de cesáreas). La historia internacional reciente lo muestra a las claras.

\footnotetext{
$\overline{\text { Correspondencia }}$

Carlos Campillo Artero

Servicio de Salud de las Islas Baleares

Palma de Mallorca

carlos.campillo@ibsalut.es
}

La fracción clínicamente injustificada del aumento secular del porcentaje de cesáreas (y aun más de las programadas) sigue poniendo en evidencia la paresia de aquellos de quienes depende poner coto a esta tendencia en apariencia imparable. Este aquellos es como el viejo adagio de Hacienda: somos todos. Además, desde los prismas de la efectividad, la seguridad y la eficiencia, continúa justificando que se desplieguen con firmeza y de una vez por todas dos conjuntos de actuaciones y mantenerlas cuanto sea necesario. Primero, conocer bien su magnitud y sus causas, no de forma puntual, sino en periodos razonables y en todos los hospitales. (Hay diversas causas de inadecuación y, por consiguiente, su reducción depende de que se incida específicamente en cada una de ellas.) Segundo, actuar, también de forma sostenida, para reducir no el porcentaje de cesáreas per se sino el de las que no deberían efectuarse a la luz de indicaciones (para las urgentes y las programadas) fundamentadas en pruebas científicas, cuyo respaldo clínico aumenta, si bien con excesiva lentitud.

Respecto al conocimiento del problema, con independencia de los estudios aislados e incompletos realizados, las aproximaciones más sólidas a la sobreindicación de las cesáreas pueden dividirse grosso modo en tres grupos. Los atlas de variaciones de la práctica médica son sumamente útiles para detectar variabilidad de las tasas entre diferentes áreas geográficas, ser el primer paso 
y sugerir - no confirmar de entrada- sobre e infrautilización (injustificadas) de esta intervención ${ }^{3}$. En otros casos, además de las tasas brutas, se estiman retrospectivamente los porcentajes de (in)adecuación de las cesáreas urgentes y programadas a indicaciones establecidas y estándares fijados por amplio consenso $0^{4,5}$. Otro estudio consiste en una intervención pre-post de aumento de la adecuación, en marcha en España desde 2006 , en la cual actualmente participan 48 hospitales públicos cuya publicación se encuentra en revisión.

El tercer grupo comprende estudios que estiman directamente sobreutilización (son, en esencia, estudios de prescripción-indicación). Entre ellos sobresalen, por citar dos ejemplos, los que realizan en la Bloomberg School of Public Health (The Johns Hopkins University). A partir de datos administrativos, como los de Medicare, y de una lista de tecnologías potencialmente sobreutilizadas, construyen índices simples y agregados, estandarizados y ajustados por riesgo y delinean patrones sistemáticos de sobreutilización de dichas tecnologías en áreas y regiones ${ }^{6}$. El segundo ejemplo es el estudio de Librero y colaboradores, que se publica en este número de la Revista Española de Salud Pública ${ }^{7}$.

El estudio de Librero ha de ser bienvenido por su sagacidad, solidez, utilidad y por descollar en un panorama caracterizado por la escasez de este tipo de evaluaciones en España. Insertado en el tercer grupo enumerado, calcula, para el conjunto de todos los partos en los hospitales públicos de la Comunidad Valenciana entre enero de 2005 y junio de 2010, el porcentaje de cesáreas en el subgrupo de mujeres de bajo riesgo de esta intervención (definido como mujeres parturientas menores de 35 años, sin embarazo múltiple ni antecedente registrado de cesárea, con gestación entre las 37 y las 41 semanas, feto en presentación cefálica y peso normal). Aunque el porcentaje de embarazos de bajo riesgo fue notable $(58,4 \%)$, los de cesáreas total y en mujeres embarazadas de bajo riesgo fueron, respectivamente, $24,4 \%$ (recorrido: de 16,3 a $41,2 \%$ ) y $11,9 \%$ (de 7 a 28,9\%). Pocas dudas respecto a sobreutilización de este procedimiento. Este indicador es útil: varía más que el porcentaje total de cesáreas, la correlación lineal entre ambos es alta y con un registro y una codificación adecuados — no siempre garantizables a priori-su monitorización no es excesivamente engorrosa.

Respecto a las actuaciones para reducir su magnitud y - lo más importante - sus consecuencias (complicaciones y gasto evitables) destacan dos tipos diferenciables por una característica intrínseca, no por la indiferencia que ambas parecen provocar a juzgar por su baja frecuencia y la imagen moteada y rala que ofrece su distribución geográfica.

Las primeras son las intervenciones aisladas y dirigidas a un solo objetivo. Las segundas son multifacéticas por cuanto incluyen diversas acciones, realizadas simultáneamente y encaminadas a diferentes objetivos, aunque uno distintivo es la modificación de comportamientos y el cambio cultural local: formación de personal sanitario y pacientes, consenso de indicaciones basadas en pruebas científicas, adopción de guías de práctica comunes, asignación de recursos necesarios, evaluación pre-post implantación de medidas de mejora y auditorías periódicas, segunda opinión obligatoria, información y retroalimentación, coordinación y gestión de equipos, fomento del liderazgo, cambios en la política institucional de los hospitales, incentivos económicos y no económicos (difusión pública de la comparación de los resultados de los hospitales, concesión de permisos para docencia, apoyo a la investigación, aumento de los márgenes de autogestión, entre otras $)^{5,8-14}$ así como la investigación realizada en 48 hospitales públicos españoles antes citada. 
Numerosos estudios, incluido el documento de consenso publicado en marzo de este año por The American College of Obstetricians and Gynecologists, acumulan pruebas científicas que muestran la falta de efectividad de las primeras para producir los cambios estructurales, de contexto, gestión y de comportamiento suficientes para reducir sustancialmente la inadecuación de las indicaciones de las cesáreas 9 . Por otro, acopian progresivamente pruebas sobre la efectividad de las multifacéticas para conseguir descensos, cuando menos aceptables, de los porcentajes de cesáreas, así como de las complicaciones y del gasto (evitable) asociados con ellas, cuya magnitud ayuda a vislumbrar la de su coste de oportunidad en cada sistema de salud ${ }^{8-14}$.

En España tenemos atlas de variaciones de cesáreas, algún estudio como el de Librero et al. ${ }^{7}$ y alguna intervención multifacética en marcha a escala nacional. Pero son hechos lamentablemente aislados y sin garantía de continuidad por lo que se explica a continuación.

No es ocioso insistir en la imperiosa necesidad de mejorar y mantener sistemas de información fiables. Librero y colaboradores lo hacen, como lo hicieron en su primer estudio de variaciones de los porcentajes de cesáreas publicado en $2000^{15}$, así como en la cortapisa que suponen para estos estudios las deficiencias en el registro de variables imprescindibles y en su codificación. Registrar bien y mantener registros clínicos depurados demanda recursos a pie de cama que siguen escaseando en nuestro país. No es baladí seguir haciendo hincapié en la necesidad de realizar evaluaciones periódicas, pre-post intervenciones de mejora y con grupo control si es posible. Ni es fútil recordar que las intervenciones enumeradas consumen recursos y exigen inversión y apoyo institucional real, suficiente y permanente.

Seguir evaluando e interviniendo poco, aisladamente y sin respaldo científico o, por el contrario, no evaluar, no intervenir y dejar las cosas cómo están es lo más cómodo pero me- noscaba indefectiblemente la efectividad, la seguridad y la eficiencia sistémicas, lo cual, por su naturaleza y magnitud, valorado desde la perspectiva poblacional, también vulnera fundamentos de la ética médica y del profesionalismo. Confiar en el voluntarismo es un craso error. $\mathrm{Su}$ comportamiento es isotópico: tiene su propio periodo de desintegración.

\section{BIBLIOGRAFÍA}

1. Goodman SN. Toward evidence-based medical statistics. 1: The $\mathrm{p}$ value fallacy. Ann Intern Med. 1999;130(12):995-1004.

2. World Health Organization. Technical consultation on Guidelines for monitoring the availability and use of obstetric services. Geneva: WHO//UNFPA/UNICEF/AMDD; 2006.

3. Bernal Delgado E, Villaverde Royo MV, Abadía Taira MB, Librero López J, Peiró S, Ridao López M. Variaciones en la utilización de cesárea en función del riesgo obstétrico en los hospitales de agudos del sistema nacional de salud. Zaragoza: Instituto Aragonés de Ciencias de la Salud; 2009.

4. Robson MS, Scudamore IW, Walsh SM. Using the medical audit. Cycle to reduce cesarean section rates. Am J Obstet Gynecol. 1996;174:199-205.

5. Calvo A, Campillo C, Juan M, Roig C, Hermoso JC, Cabeza PJ. Effectiveness of a multifaceted strategy to improve the appropriateness of cesarean sections. Acta Obstet Gynecol Scand. 2009;88(7):842-5.

6. Segal SJ, Bridges JF, Chang HY, Chang E, Nassery N, Weiner $\mathrm{J}$, et al. Identifying possible indicators of systematic overuse of health care procedures with claims data. Med Care. 2014;52(2):157-63

7. Librero J, Peiró S, Belda A, Calabuig J. Porcentaje de cesáreas en mujeres de bajo riesgo: un indicador útil para comparar hospitales que atienden partos con riesgos diferentes. Rev Esp Salud Pública. 2014;88(3):postprint. Disponible en: http://www.msssi.gob.es/biblioPublic/publicaciones/recursos_propios/resp/revista_cdrom/vol88/vol 88_3/JLL.pdf

8. Cahillet N, Dumont A. Evidence-based strategies for reducing cesarean section rates: a meta-analysis. Birth 2007;34(1):53-64.

9. The American College of Obstetricians and Gynecologists. Save prevention of the primary cesarean delivery. Obstetric care consensus. Washington, DC: ACOG; 2014 
10. Walker R, Turnbull D, Wilkinson C. Strategies to address global cesarean section rates: A review of the evidence. Birth 2002;29(1):28-39.

11. Grimshaw JM, Shirran L, Thomas R, Mowatt G, Fraser C, Bero L, et al. Changing provider behavior. An overview of systematic reviews of interventions. Med Care. 2001;39:II-2-45.

12. Bloomfield T. Caesarean section. NICE Guidelines and management of labour. J Obstet Gynaecol. 2004;24(5):485-90.

14. Mazzocco K, Petitti DB, Fong KT, Bonacum D, Brookey J, Graham S, et al. Surgical team behaviours and patient outcomes. Am J Surg. 2009;197(5):678-85.

15. Trowbridge R, Weingarten S, Wachter RM, Markowitz AJ. Educational Techniques used in changing provider behavior. In: Shojania KG, Duncan BW, McDonald KM, editors. Making health care safer: a critical analysis of patient safety practices. Evidence Report/Technology Assessment No. 43 (Prepared by the University of California at San Francisco-Stanford Evidence-based Practice Center under Contract No. 290-97-0013), AHRQ Publication No. 01-E058. Rockville, MD: Agency for Healthcare Research and Quality; 2001:595-9.

16. Librero J, Peiró S, Calderón SM. Interhospital variations in caesarean sections. A risk adjusted comparison in the Valencia public hospitals. J Epidemiol Community Health. 2000;54(8):631-6. 